\title{
An Automated Stable Personalised Partner Selection for Collaborative Privacy EDUCATION
}

\author{
Evans Girard ${ }^{1}$, Rita Yusri $^{1}$, Adel Abusitta ${ }^{2}$ and Esma Aïmeur ${ }^{1}$ \\ ${ }^{1}$ University of Montreal, Montreal, Canada \\ ${ }^{2}$ McGill University, Montreal, Canada
}

\begin{abstract}
E-learning platforms have never been as in-demand as they are now since the recent pandemic making privacy education more important than ever. However, for the most part, these platforms are single-user learning environments and lack student-student interactions. To overcome this deficiency, we propose a collaborative e-learning platform for privacy education that matches students in a stable and automatic manner according to students' preferences. Each student is represented by a vector profile that is created from behavioural skills and academic knowledge obtained from the platform. Once the preferences are determined, the residents-hospitals matching algorithm is applied to select students who will collaborate with one another. Experimental results show that the proposed model offers an effective way to create stable, thus satisfied, coalitions of students from two groups of arbitrary sizes. In addition, the automation allows students to skip the tedious process of manually selecting partners. Therefore, saving their time to collaborate on privacy education with their teammates helping them to increase their privacy awareness.
\end{abstract}

\section{KEYWORDS}

Privacy Education, Collaborative Learning, E-learning, Matching Game

\section{INTRODUCTION}

It is increasingly obvious that everyday the dependence on our cyber-life exposes us to privacy issues. This is especially true for youth since they spend a large part of their life in the cyber-space. Therefore, privacy education should be an important component of general education in order to highlight the importance of privacy and the risks of certain habits or behaviours [1]. Privacy elearning platforms become an essential tool to help teenagers become more privacy-aware [2], [3].

Several e-learning platforms have been proposed in the literature (e.g., [4], [5], [6] [7], [8], [9], [10], [11]). These platforms, however, lack of student-to-student interactions since they offer single-user learning environments [3] [12] [13]. Traditional e-learning platforms designed for privacy education are also not well suited for student-to-student interactions. In parallel, Computer-supported collaborative learning (CSCL) has become a set of crucial pedagogical techniques giving many advantages by using collaborative learning [14], [15], [4], [7], [16], [17]. Collaborative learning allows students to cooperate and exchange feedback and information [18] [19]. This in turn enables students to better understand course materials and achieve mutual benefits [13] [3]. However, one major drawback in this type of environment is the lack of mechanisms to group partners in an optimal way. Matching incompatible individuals can have significant negative impacts such as preventing a student from focusing and achieving the main goals of cooperation [3]. Therefore, selecting optimal partners in collaborative learning is essential to achieve better learning outcomes. 
The above-mentioned problems have been addressed by two platforms: Teens-Privacy [20] and Teens-Online [3]. The first platform allows, in the first place, to extend traditional e-learning platforms to include a collaborative environment. The second platform uses a stable marriage algorithm to match students thus addressing the lack of mechanisms to group students in the CSCL paradigm [3]. Both platforms make use of Academic and Socio-psychological factors constructed by the means of quizzes to create the preference lists of each student needed to run the matching algorithm. More precisely, each student has to answer quizzes to build or complete a profile. Once the profile is completed, each student has to rank students of the other group in order of preference. This ranking (list of preferences) becomes the input for the matching algorithm. However, this process can be tedious and become a burden for students.

This paper is an improvement of the previous work proposed in [20] and [3], where we present an automated stable personalised partner selection for collaborative Privacy Education. In the proposed framework, we include a new component to automate the process of selection by the mean of similarity measures. This can be done without disallowing the possibility of manually selecting partners. The automation process can be used to bootstrap the initialization phase or be used at any other time if needed. It thus offers the possibility to fully or partially automate the selection process. It is worth mentioning here that the stable marriage matching algorithm in use on the previous platforms (i.e., [20] and [3]) requires the two groups be the same size. Since, this requirement is restrictive we adopted the extended version of the Gale-Shapley stable marriage algorithm: the resident-hospital algorithm that allows us to match groups of unequal sizes.

In the proposed framework, we consider two categories as the selection criteria, which are based on the recent classification done by [21]. The first category is Academic factors, which consist of the topic-specific knowledge level of students. The advantage of using these factors is to enhance the benefits of students' collaboration by pairing students who can help each other based on complementary competences [20]. The second category is Socio-psychological factors, represented by behavior factors, which consist of personality traits that are considered useful to improve collaborative work and help students to participate effectively [21]. In summary, this paper consists of the following contributions:

- Devising an efficient and stable personalized-based partner(s) selection algorithm for collaborative learning. The proposed algorithm allows us to match groups of unequal sizes, which improves the flexibility of collaborative e-learning.

- Integrating a Residents-Hospitals algorithm into the proposed collaborative e-learning platform in order to guarantee the selection of partners is done automatically.

The rest of this paper is organized as follows. In Section 2, we discuss the related work. Section 3 formulates the proposed framework. In Section 4, we present our empirical results to evaluate performance of the proposed framework. Lastly, Section 5 concludes the paper and presents future research.

\section{BACKGROUND}

Several e-learning environments and platforms have been proposed in the last few years (e.g., [4], [7], [8], [9], [10], [11]). The main disadvantage of these approaches is that they do not support student-to-student interactions since they offer single-user learning environments. There is also a large number of studies on collaborative learning showed the benefits of such environments on 
students (e.g., [22], [16], [23],[24], [25]). Also several studies (e.g., [26], [27], [28], [29]) have been proposed in the context of Computer-Supported Collaborative Learning (CSCL). However, the lack of methods for selecting the optimal partner(s) is evident in these frameworks. To overcome the situation, this paper proposes a matching algorithm deemed to be stable from the field of game theory: Residents-Hospitals (RH) matching algorithm [30]. This algorithm is useful and famous since it is behind The National Resident Matching Program (NRMP) in the United States of America, Canada and elsewhere [31]. This program is used to place medical students into residency training programs. The algorithm is an extended version of the stable marriage problem [32]. Indeed, the stable marriage problem restricts the two groups to be matched to be of equal size which is not the case in the extended version. The algorithm uses preferences lists to build the matching. Concretely, each student must rank students from the other group according to their preference. In our e-learning platform, we propose a framework that matches students from two distinct groups of unequal sizes by automatically building preferences list by means of similarity measures and then running RH. Such an approach leads to a stable matching of students. That is, no student has an incentive to leave her/his current partner(s) and pair with another one.

In fact, there are many important studies in the field of CSCL and collaborative problem solving (e.g., [25], [33], [34]). There are also several studies that use students' individual characteristics and preferences as grouping criteria (e.g., [35], [36], [37], [38], [39]). The limitation of these approaches in the collaborative environment is the lack of mechanisms for choosing the optimal partners that help students engage in learning activities. One should note that working with an incompatible student may negatively impact the learning process. Some researchers indicate that inadequate peer matching has been the main reason for many unsuccessful collaborative learning applications [40]. Thus, optimal partner selection is a critical fundamental process and should be done in a proper way [41] [42].

To address the above-mentioned problem, we propose a collaborative e-learning platform for privacy education that matches students in an automatic and stable manner. The matching procedure is performed according to students' preferences. To this end, each student is represented by a vector profile, which is extracted from behavioural skills and academic knowledge. The proposed framework is integrated with a Residents-Hospitals algorithm in order to ensure that the selection process (i.e., selection of partners) is done automatically.

\section{THE PROPOSED FRAMEWORK}

Figure 1 shows the proposed framework. As can be seen in Figure 1, the inputs consist of two groups of students, which are transformed into vectors. Thereafter, a similarity score such as Jaccard, Cosine and Dice is applied by comparing every vector of each group with every vector of the other group. These lists of scores are then ordered from highest to lowest (most similar to least similar). These are considered to be the preferences lists. Finally, the matching algorithm is run.

\subsection{Student Profile}

Privacy-specific quizzes, forms, reports, exams and such provide valuable information (knowledge, behaviours characteristics) about a particular student. When this information is collected in a similar fashion for a set of students, it can be used to better know a student and even compare different students altogether. In particular, the "Teens-Privacy" platform accumulates such information [20]. From the system's perspective, we propose to structure a student profile in the form of a vector. All these vectors (students) are part of a vector space $V_{s} \subseteq \mathbb{R}_{+}^{n}$ that allows us to perform mathematical 


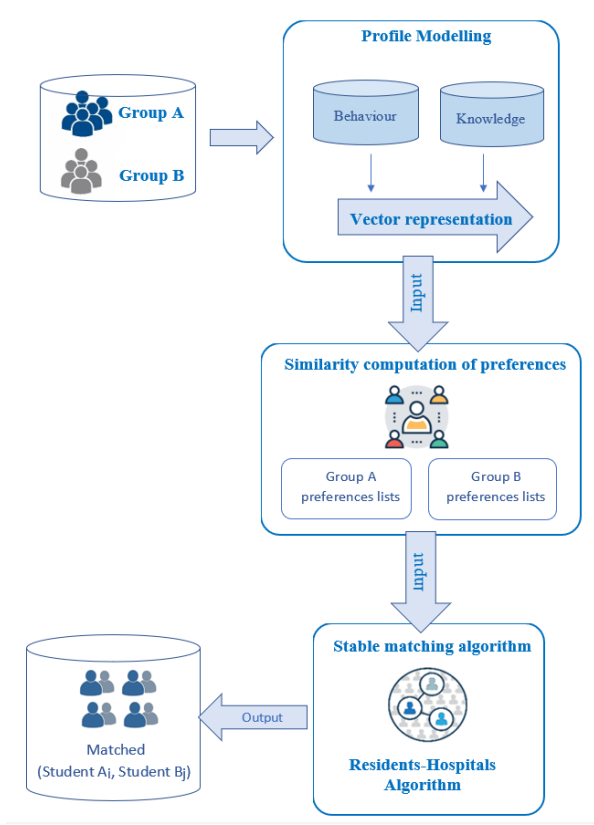

Figure 1: Framework of the matching module

operations in order to add automation to the system. Each student is therefore represented as a vector of numerical values inferred from knowledge gathered, behaviours and skills. Formally, let's assume that all these $m \in \mathbb{N}$ results $r_{i}$ form a sequence $\left(r_{1}, r_{2}, \ldots, r_{m}\right)$ representing all the system's knowledge about students. From this sequence, a vector space basis can be derived. The ordering of the results is arbitrary but must remain static once chosen and every student must complete all the same quizzes, exams, forms, etc for consistency.

\subsubsection{Example}

Suppose two groups $G_{1}=\{$ Josh, Alice, Bob $\}, G_{2}=\{$ Zoe, Jason $\}$ of students are asked to complete two quizzes. One quiz asks how important these characteristics are to them (conscientiousness, gregariousness, independence) and a second one based on knowledge asks what is their level of competence in $\{$ security, privacy\}. All quizzes are rated on a scale from 0 to 10. Let's create a labelling such that $r_{1} \leftarrow$ conscientiousness, $r_{2} \leftarrow$ gregariousness, $r_{3} \leftarrow$ independence, $r_{4} \leftarrow$ security, $r_{5} \leftarrow$ privacy. In the end, let's suppose an arbitrary rating that led: $S_{\mathrm{Josh}}=(1,1,1,1,1), s_{\text {Alice }}=$ $(2,6,2,7,3), s_{\mathrm{Bob}}=(5,6,9,7,3), s_{\mathrm{Zoe}}=(2,2,2,10,10), s_{\mathrm{Jason}}=(8,6,8,7,5)$

\subsection{Profile Structural Particularity}

"Teens-Privacy" framework separates the profile (or the students space at this point) into 2 distinctive categories: one including the knowledge factors and the other one the behaviour factors. In our model, the first $k<m$ elements could represent the results attached to the knowledge factors while the remaining axis represents the behavioral ones. The critical point is that in the implementation, we need to know which of the two categories a given axis belongs in. As we will describe later on in this article, this distinction is needed in order to select students with complementary skills. 


\subsection{Partner Selection}

In our framework that lies on the core concept of game theory, every student tries to maximize his or her utility when associating with students of the other group. In particular, "Teens-Privacy", as described in the previous paper, takes advantage of the stable marriage algorithm to match students creating a fully-cooperative framework [20]. This algorithm requires that the two groups of students be of the same size. However, an extended version of this algorithm that can accommodate groups of unequal sizes exists: residents-hospitals matching algorithm. We will upgrade the previous framework with this version instead. It should be stressed that with this version the framework is a cooperative framework. This is due to the fact that if some students were not matched in a given period, they can be matched in the future periods as long as the students' preferences lists are being changed over time.

In order to use matching algorithms, preferences lists must be constructed for every student. In "Teens-Privacy", this process is done manually. We suggest the possibility to automate this part when needed with the use of similarity measures between student vectors.

\subsection{Automation of the Preferences list}

Each student $s$ has to rank students of the other group in order of strict preferences resulting in a particular preference list $\mathcal{P}_{S}$. Given two disjoint groups of students $G_{i}, G_{-i} \subset V_{S} \neq \varnothing$ the preference list $\mathcal{P}_{S}$ of a given student $s$ in group $G_{i}$ is a permutation $\sigma: G_{-i} \rightarrow G_{-i}$ of $G_{-i}$ that can be written as $\mathcal{P}_{s}=\sigma\left(G_{-i}\right)$ and similarly for $G_{i}$. Moreover, if $s$ strictly prefers $x$ over $y$, we write $x>_{s} y$.

We will assume that a student would prefer a peer that have similar behavioural characteristics but complementary academic knowledge over another one. Indeed, it is not illogical to think that two persons sharing similar behaviours will fit together while having complementary knowledge will benefit everybody. Therefore, building preferences is analogous to building similarities. The idea to automate the creation of preferences lists consists in measuring the similarity of two student vectors to obtain a score. This score is used for ordering. A better score for $s_{a}$ than $s_{b}$ means that $s_{a}>_{s} s_{b}$. Moreover, to make sure that two students have complementary academic skills a transformation $T: V_{S} \rightarrow V_{S}$ is applied to the student creating his/her preferences list such that components representing the academic skills are changed for their complement (for instance, if the component skills in security is $6 / 10$ then its complement becomes $4 / 10$ ). This ensures that someone with a specific strong component will artificially become similar to a weak one in this activity and vice-versa.

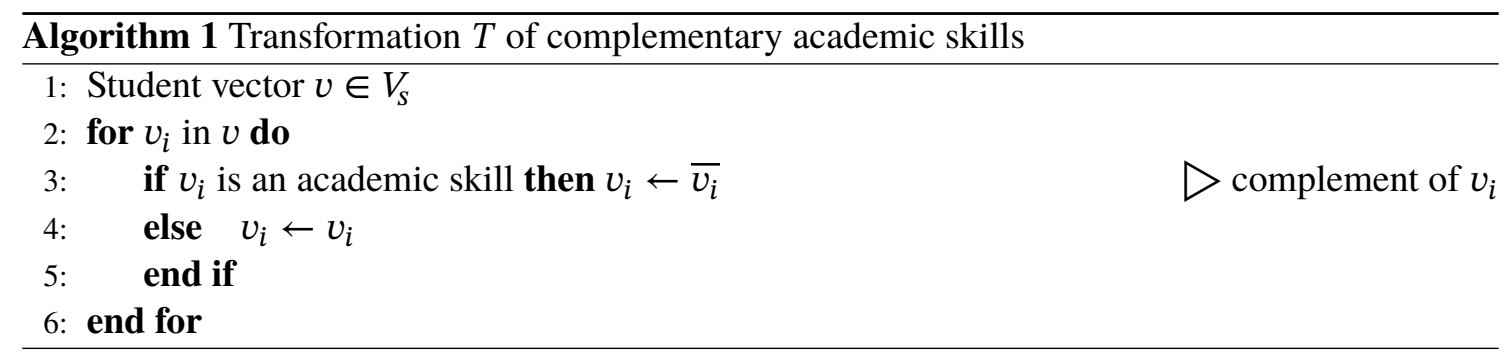

\subsubsection{Example}

Let's take Josh's profile from the last example. Since $r_{4}$ and $r_{5}$ are knowledge-specific components than the transformation leads to: 
$S_{\text {Josh }}^{\prime}=(1,1,1,10-1,10-1)=(1,1,1,9,9)$ Our strategy is to construct complete preferences lists (each student orders all students of the other group) but, if needed, one could build partial lists. For instance, one could design a test function that takes into account only students having a score above a given threshold.

\subsubsection{Similarity Measures}

The literature is abundant about similarity measures and there does not exist a single abstract definition for them but it is sufficient to say that a similarity measure is a real-valued function that indicates how "close" are two mathematical entities are from each other. To compute similarity in this article, two well-known similarity measures[43] $\operatorname{sim}: V_{S} \times V_{S} \rightarrow \mathbb{R}$ will be used: Jaccard, $J$ and cosine, cos defined by

$$
\cos (X, Y) \equiv \frac{X \cdot Y}{\|X\|\|Y\|} ; J(X, Y) \equiv \frac{X \cdot Y}{\|X\|^{2}\|Y\|^{2}-X \cdot Y} \quad X, Y \in V_{s}
$$

Where $\cdot$ is the inner product of two vectors and $\|\cdot\|$ is the modulus or length of a vector.

Given two disjoint sets (of students) $A, B$, the pseudo-code to generate the preference list of students in $B$ for a particular student $s_{a}$ in $A$ is:

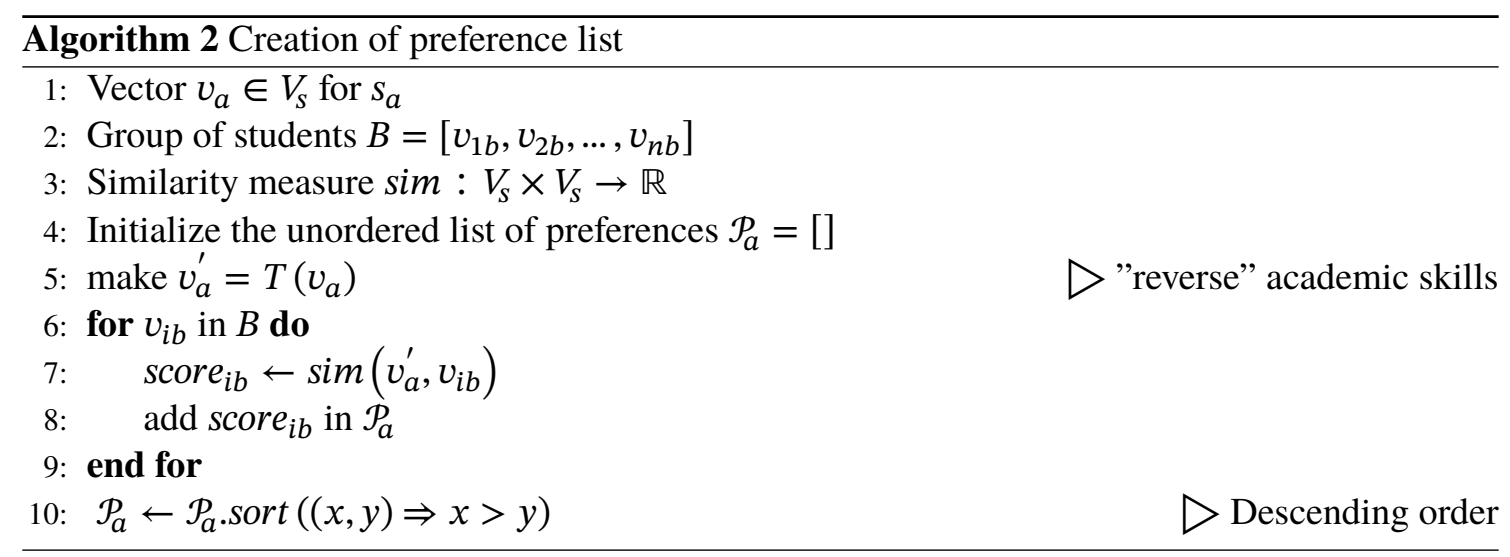

\subsubsection{Example}

For Josh, the Jaccard coefficients in relation to group $G_{2}$ are: $J\left(s_{\text {Josh }}^{\prime}, s_{\text {Zoe }}\right) \approx 0.974 J\left(s_{\text {Josh }}^{\prime}, s_{\text {Jason }}\right) \approx$ 0.476 . Which means that Zoe $>_{\text {Josh }}$ Jason and therefore $\mathcal{P}_{\text {Josh }}=$ (Zoe, Jason) because for the Jaccard index values closer to 1 have a higher degree of similarity. Indeed, Josh; and Zoe value the behavioural characteristics similarly but Zoe has better academic skills making Zoe a very good potential match for Josh thus Zoe is placed highest in importance in the preference list. Repeating the same procedures for every student we obtain: $\mathcal{P}_{\text {Josh }}=($ Zoe, Jason $), \mathcal{P}_{\text {Alice }}=($ Zoe, Jason $), \mathcal{P}_{\text {Bob }}=$ (Jason, Zoe $), \mathcal{P}_{\text {Zoe }}=($ Josh, Bob, Alice $), \mathcal{P}_{\text {Jason }}=($ Bob, Alice, Josh $)$. Note that if two classmates have equal components thus having the same similarity value, then it is up to the implementation to determine which student is the most preferred. In other words, who comes first in the rankings (strict preferences). 


\subsection{Matching}

Once preference lists are built for every student, the next step is to match them based on these lists. In our previous article the Gale-Shapley Stable Matching algorithm (SM) was used. Since this algorithm requires two groups with the same number of students, we would like to use a more general approach allowing for two groups of different sizes. A natural extension is to use the classical Resident-Hospital assignment problem $(\mathbf{R H})$. It can be viewed as a game with two sets of players whose aim is to assign one group to the other one based on preferences. Therefore, the game can be defined by the tuple $(\mathcal{N}, M, \mathcal{P})$ where $\mathcal{N}$ are the players, $M$ every possible matchings or actions and $\mathcal{P}$ the preference profile. To detail this game we will use two distinct sets of players $R=\left\{r_{1}, \ldots, r_{n}\right\}$ and $H=\left\{h_{1}, \ldots, h_{m}\right\}$ where $m, n \in \mathbb{N}$ called the residents and the hospitals respectively to be consistent with the terminology found in the literature. Each member $h_{i} \in H$ has a quota or capacity $q_{i} \in \mathbb{N}-\{0\}$ indicating how many $r \in R$ it can accept. The rules are: 1$)$ each $r \in R$ must rank in strict order the hospitals $H^{\prime} \subseteq H$ he/she is willing to accept $\mathcal{P}_{r}=\sigma\left(H^{\prime}\right)$; 2) each hospital $h \in H$ must rank in strict order all the residents that have ranked it. Therefore, given the sets $D_{h}=\left\{r \in R \mid h \in \mathcal{P}_{r}\right\}$ each ranked hospital must create its preference list $\mathcal{P}_{h}=\sigma\left(D_{h}\right)$

Besides, the pair $\left(r_{i}, h_{j}\right) \in R \times H$ is an assignment deemed acceptable if $h_{j} \in H$ is in $r_{i}$ 's preference list and vice-versa. The solution of an instance of the game is called a matching $M$. Formally, if $A$ is the set of all acceptable pairs, then $M: R \rightarrow R \times H$ where $M \subseteq A$. The notation for all pairs containing $r_{i}$ in the matching is $M\left(r_{i}\right)=\left\{\left(r_{i}, h_{j}\right) \in A\right\}$. A matching can possibly leave some residents unassigned. Precisely, to be a valid matching

- $\left|M\left(r_{i}\right)\right| \leq 1 \forall r_{i} \in R$ (no resident in more that one pair).

- $\left|M\left(h_{i}\right)\right| \leq q_{i} \forall h_{i} \in H$ (no hospital appears in more pairs than its quota).

If $M$ contains no blocking pair then it is considered stable. A blocking pair $\left(r_{i}, h_{j}\right) \in A \backslash M$ is defined as $h_{j}>_{r_{i}} M\left(r_{i}\right)$ and $r_{i}>h_{j} M\left(h_{j}\right)$. In short, it means that every agent in the blocking pair would prefer to be assigned to one another than their current matching. Every instance of this game admits a stable matching that can be found in linear time. Preference lists need not be complete to use RH.

In this article, we will use the resident-optimal stable matchings meaning that each $r_{i}$ that is assigned is matched with the best hospital he/she can obtain in a stable matching. Moreover, every unassigned $r_{k}$ is unassigned in all stable matchings. Before presenting the algorithm we apply some restrictions to ensure that all students are matched. The first restriction we impose is that preference lists for every student of both groups must be strict and complete (all students of the other groups are ranked). We cover only the case where there is an equal or superior number of $r \in R$. Thus, the largest group should always be the "residents" group. In addition, we want to compute a quota large enough to welcome everybody (unmatched students can still exist). Mathematically, $|R|>|H|$ or $|R|=|H|$ and $\mathcal{P}_{r_{i}}=\sigma(H) \forall r_{i} \in R ; \mathcal{P}_{h_{i}}=\sigma(R) \forall h_{i} \in H$ and $q_{1}=\cdots=q_{n}=q \geq 1, n=|H|$. From the pigeonhole principle and considering the residents as pigeons and the hospital's quotas as pigeonholes, then there should be enough holes if $q=\operatorname{ceil}\left(\frac{|R|}{|H|}\right)$. Or put differently, if we see a hospital having $q$ quotas as $q$ hospitals with 1 quota then we have $|H| \cdot q$ possible positions to place the residents since we restricted quotas to be uniform. Thus, $|R| \geq q \cdot|H| \Rightarrow q \leq \frac{|R|}{|H|}$. Therefore, the ceiling value of $\frac{|R|}{|H|}$ seems like a good choice for automatically determining a large enough $q$. For instance, if there are 3 residents and 2 
hospitals then the quota will be $q=\operatorname{ceil}\left(\frac{|3|}{|2|}\right)=2$ meaning that each hospital can accept up to 2 students. With these constraints in mind, we can apply the classical Resident-Hospital algorithm to our need:

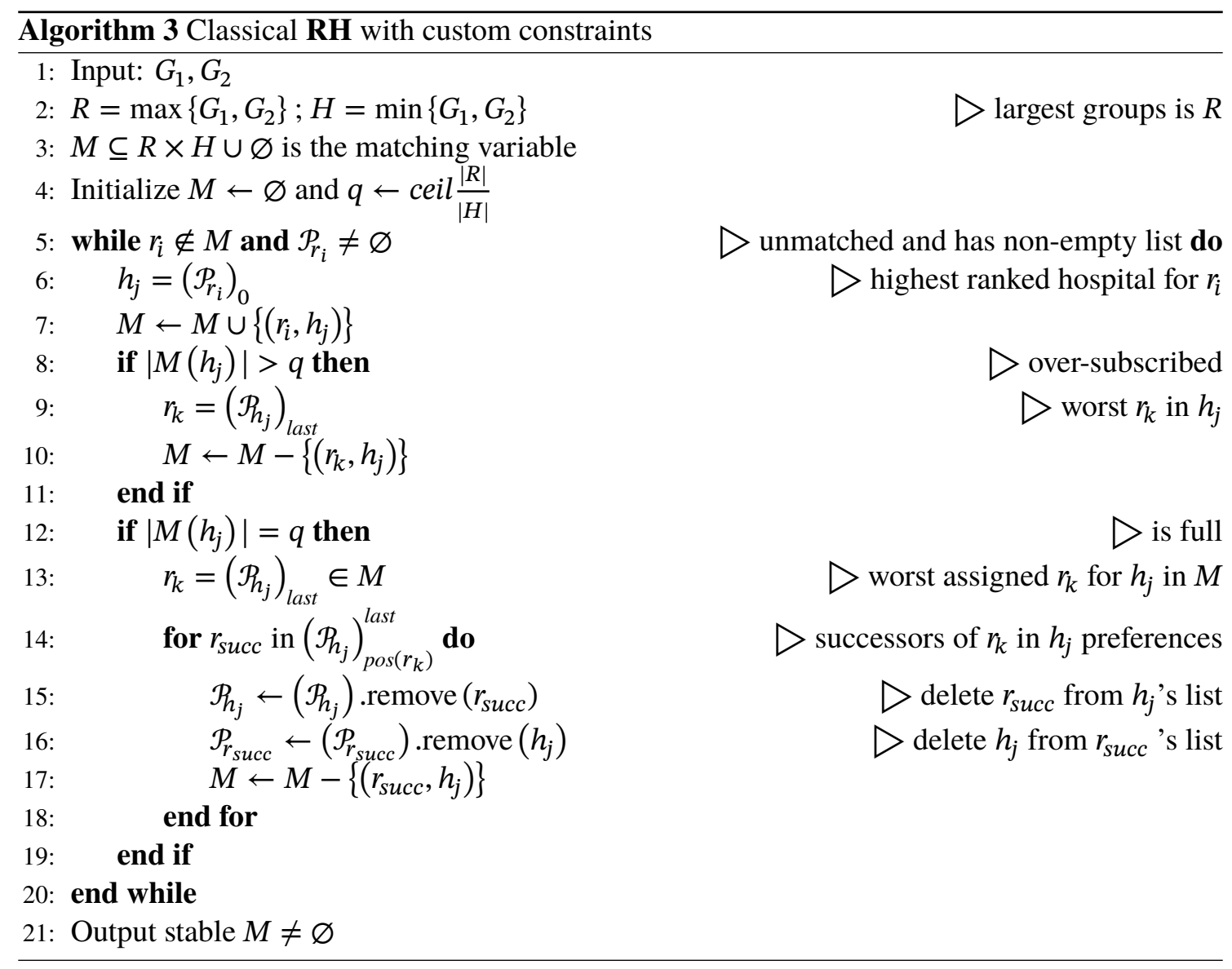

Given data from exams score, answers from quizzes and so forth about students, profiles are created. Our algorithm takes as inputs two groups A and B of students. The sizes of the groups do not need to be equal. The first step consists in the modelling of these profiles as real-valued vectors. The second step computes, for every student, a measure of similarity between him/her with the students of the other group. These measures are then ordered in descending order to represent the preferences lists. In the last step, these two groups, extended with a preferences list for every student, are given to the Gale-Shapley Residents-Hospitals matching algorithm. It outputs a stable matching that consists in the paring of a student from group A with student of group B. In the case of unequal groups, a student from group B can be matched with multiple students of group A or be unmatched.

\section{Experimental Evaluation}

We designed a proof-of-concept experiment to see to what extent the proposed algorithm can improve the satisfaction of the students. In our experiments, two groups A and B are considered. The sizes of the two groups do not need to be equal. The student profile is based on qualities and knowledge. There are $\mathbf{7}$ qualities: conscientiousness, gregariousness, independence, intuitiveness, 
kindness, passionate and respectfulness rated on a scale from 0 to 10. Similarity, there are $\mathbf{5}$ skills based on knowledge: privacy, security, technical aspects, economical aspects and regulations and legislation.

The first step consists in obtaining the profile of each student. In this experiment groups: $A$ and $B$ consist of 10 students. The second step consists in generating the preferences list of every student. As for the choice of the similarity measure, The Jaccard index was used [44]. The last step is the running of the matching algorithm $(\mathbf{H R})$. To establish the rankings, the fit score metric was adopted [21]. It compares two students by measuring how much one's qualities fit the other one's

preferences. Formally, $f i t_{b \rightarrow a}=\sum_{i=1}^{12} p_{i b} \cdot w_{i a}, \sum_{i} w_{i a}=100$ where $p_{i b} \in[0,10]$ is quality $i$ in student $b$ 's profile while $w_{i a}$ is the weight of quality $i$ and student $a$ 's profile. It is to be noted that the weights assigned to the qualities are distributed in decreasing way, the highest weight reflects the most important quality for this student.

The RH algorithm was compared with three other methods: preference-based and characteristicsbased homogeneous clustering method and the random-based matching method (RM). The preferencebased homogeneous clustering method (PCM) [45] was used to pair students with the most similar preferences together, whereas, in the characteristics-based homogeneous clustering method (CCM), students with the most similar characteristics are paired together.

\subsection{Results}

Figure 2 shows the performance of the different methods, the best performances are shown in bold. Our algorithm does not guarantee an optimal partner for every student. However, it performs better, on average, than the other methods. Moreover, we are sure that the matching is stable in the sense of game theory. 


\begin{tabular}{|c|c|c|c|c|}
\hline \multicolumn{5}{|c|}{ Fit scores for the four methods } \\
\hline & RM $[\%]$ & CCM $[\%]$ & PCM $[\%]$ & RH $[\%]$ \\
\hline A1 & 54.10 & 56.80 & 54.10 & $\mathbf{6 7 . 7 0}$ \\
\hline A2 & 65.10 & 54.90 & 65.10 & $\mathbf{7 8 . 1 0}$ \\
\hline A3 & $\mathbf{6 8 . 7 0}$ & 54.80 & 62.60 & 61.10 \\
\hline A4 & 50.80 & 61.00 & 63.20 & $\mathbf{7 8 . 8 0}$ \\
\hline A5 & 46.90 & 54.10 & 56.80 & $\mathbf{6 1 . 6 0}$ \\
\hline A6 & 66.60 & 47.00 & 54.90 & $\mathbf{7 1 . 8 0}$ \\
\hline A7 & 64.00 & 61.70 & 45.00 & $\mathbf{7 3 . 8 0}$ \\
\hline A8 & $\mathbf{7 0 . 5 0}$ & 62.60 & 69.80 & 59.10 \\
\hline A9 & 60.70 & $\mathbf{7 7 . 0 0}$ & 60.70 & 65.80 \\
\hline A10 & 48.10 & 82.60 & $\mathbf{8 1 . 5 0}$ & 78.10 \\
\hline B1 & 72.50 & 55.20 & 54.70 & $\mathbf{8 2 . 2 0}$ \\
\hline B2 & 62.80 & 62.10 & 65.00 & $\mathbf{8 3 . 3 0}$ \\
\hline B3 & $\mathbf{6 8 . 6 0}$ & 57.10 & 47.10 & 52.10 \\
\hline B4 & 59.20 & 78.60 & 66.70 & $\mathbf{8 1 . 2 0}$ \\
\hline B5 & 55.20 & 54.70 & 55.20 & $\mathbf{7 7 . 7 0}$ \\
\hline B6 & 59.90 & 65.00 & 59.90 & $\mathbf{8 5 . 5 0}$ \\
\hline B7 & 56.70 & 57.60 & $\mathbf{7 6 . 2 0}$ & 64.70 \\
\hline B8 & 48.90 & 59.80 & $\mathbf{7 3 . 4 0}$ & 55.80 \\
\hline B9 & 44.70 & 73.20 & $\mathbf{8 5 . 8 0}$ & 82.20 \\
\hline B10 & 73.70 & $\mathbf{8 6 . 6 0}$ & 73.70 & 78.10 \\
\hline
\end{tabular}

Figure 2: Results comparison of the matching model

\section{Conclusion and Future Work}

In this paper, we propose a collaborative e-learning platform for privacy education. The proposed platform allows students to obtain higher learning outcomes in a collaborative environment. This can be done by enabling them to select optimal partner(s) using the proposed partner selection algorithm, based on a stable matching algorithm. The proposed platform also provides a stable student-student matching according to their preferences, behaviour and topics needing improvement. Moreover, we integrate a Residents-Hospitals algorithm into the proposed selection algorithm in order to guarantee the selection of partners is done automatically. Experimental results show that the proposed framework outperforms other approaches in terms of enhancing the quality of selected partners and improving learning outcomes. In the future, we would like to test and integrate the proposed platform with many educational platforms (e.g., Kadenze and Moodle) and different materials (i.e., courses) such as Computer Science and History. Furthermore, we plan to improve the collaboration by making it a joint effort between the educational platforms and the students themselves. Through collaboration, educational platforms can share their knowledge and experience in order to enhance the learning outcomes of their students and achieve mutual benefits.

\section{REFERENCES}

[1] S. Egelman, J. Bernd, G. Friedland, and D. Garcia, "The teaching privacy curriculum," in Proceedings of the 47th ACM Technical Symposium on Computing Science Education, 2016, pp. 591-596. Cited on page(s): 1

[2] M. Ioanna and K. Anthi, "Children using facebook: Personality traits, self-concept and school 
performance," International Journal on Integrating Technology in Education (IJITE), vol. 9, no. 4, pp. 11-26, 2020. Cited on page(s): 1

[3] R. Yusri, A. Abusitta, and E. Aïmeur, "Teens-online: a game theory-based collaborative platform for privacy education," International Journal of Artificial Intelligence in Education, pp. 1-43, 2020. Cited on page(s): 1, 2

[4] J. Elsayed, L. Maawad, and Z. khalifa, "E-supporting performance styles based on learning analytics for development of teaching practices in teaching science," International Journal on Integrating Technology in Education (IJITE), vol. 9, no. 2/3, pp. 1-11, 2020. Cited on page(s): 1,2

[5] M. C. Maphalala and O. T. Adigun, "Academics' experience of implementing e-learning in a south african higher education institution." International Journal of Higher Education, vol. 10, no. 1, pp. 1-13, 2021. Cited on page(s): 1

[6] C. De Medio, C. Limongelli, F. Sciarrone, and M. Temperini, "Moodlerec: A recommendation system for creating courses using the moodle e-learning platform," Computers in Human Behavior, vol. 104, p. 106168, 2020. Cited on page(s): 1

[7] L. Hanna, D. Barr, H. Hou, and S. McGill, "An exploration of the relationship between modern foreign language $(\mathrm{mfl})$ teachers and computer assisted language learning (call)," International Journal on Integrating Technology in Education (IJITE), vol. 9, no. 4, pp. 1-11, 2020. Cited on page(s): 1, 2

[8] G. Yaw Koi-Akrofi, E. Owusu-Oware, and H. Tanye, "Challenges of distance, blended, and online learning: A literature based approach," International Journal on Integrating Technology in Education (IJITE), vol. 9, no. 4, pp. 27-39, 2020. Cited on page(s): 1, 2

[9] A. P. Sethi and R. Subramoniam, "Use of technology in education, but at what cost?" International Journal on Integrating Technology in Education (IJITE), vol. 8, no. 1, pp. 13-18, 2019. Cited on page(s): 1, 2

[10] M. Alshayeb, "Enhancing student's learning and satisfaction through the use of social media," International Journal on Integrating Technology in Education (IJITE), vol. 7, no. 2, pp. 1-12, 2018. Cited on page $(s): 1,2$

[11] N. Hasany, "E-learning student assistance model for the first computer programming course," International Journal on Integrating Technology in Education (IJITE), vol. 6, no. 1, pp. 1-7, 2017. Cited on page(s): 1, 2

[12] D. Al-Fraihat, M. Joy, J. Sinclair et al., "Evaluating e-learning systems success: An empirical study," Computers in Human Behavior, vol. 102, pp. 67-86, 2020. Cited on page(s): 1

[13] F. A. Orji, K. Oyibo, R. Orji, J. Greer, and J. Vassileva, "Personalization of persuasive technology in higher education," in Proceedings of the 27th ACM Conference on User Modeling, Adaptation and Personalization, 2019, pp. 336-340. Cited on page(s): 1

[14] A. Halavais, "Computer-supported collaborative learning," The International Encyclopedia of Communication Theory and Philosophy, pp. 1-5, 2016. Cited on page(s): 1 
[15] N. T. Heffernan and C. L. Heffernan, "The assistments ecosystem: Building a platform that brings scientists and teachers together for minimally invasive research on human learning and teaching," International Journal of Artificial Intelligence in Education, vol. 24, no. 4, pp. 470-497, Dec 2014. [Online]. Available: https://doi.org/10.1007/s40593-014-0024-x Cited on page(s): 1

[16] M. Awedh, A. Mueen, B. Zafar, and U. Manzoor, "Using socrative and smartphones for the support of collaborative learning," International Journal on Integrating Technology in Education (IJITE), vol. 3, no. 4, pp. 17-24, 2014. Cited on page(s): 1, 3

[17] D. Zapata-Rivera, "Open student modeling research and its connections to educational assessment," International Journal of Artificial Intelligence in Education, Oct 2020. [Online]. Available: https://doi.org/10.1007/s40593-020-00206-2 Cited on page(s): 1

[18] M. L. Petrunich-Rutherford and F. Daniel, "Collaborative quizzes: Impact on student performance and attendance," Teaching of Psychology, vol. 46, no. 2, pp. 115-120, 2019. Cited on page $(s): 1$

[19] T. J. Nokes-Malach, J. E. Richey, and S. Gadgil, "When is it better to learn together? insights from research on collaborative learning," Educational Psychology Review, vol. 27, no. 4, pp. 645-656, 2015. Cited on page(s): 1

[20] R. Yusri, A. Abusitta, and E. Aïmeur, "A stable personalised partner selection for collaborative privacy education," in Adjunct Publication of the 28th ACM Conference on User Modeling, Adaptation and Personalization, 2020, pp. 47-52. Cited on page(s): 2, 3, 5

[21] T. N. Thanh, M. Morgan, M. Butler, and K. Marriott, "Perfect match: Facilitating study partner matching," in Proceedings of the 50th ACM Technical Symposium on Computer Science Education, 2019, pp. 1102-1108. Cited on page(s): 2, 9

[22] E. Aimeur and C. Frasson, "Analyzing a new learning strategy according to different knowledge levels," Computers \& Education, vol. 27, no. 2, pp. 115-127, 1996. Cited on page(s): 3

[23] T. Monahan, G. McArdle, and M. Bertolotto, "Virtual reality for collaborative e-learning," Computers \& Education, vol. 50, no. 4, pp. 1339-1353, 2008. Cited on page(s): 3

[24] K. Franceschi, R. M. Lee, S. H. Zanakis, and D. Hinds, "Engaging group e-learning in virtual worlds," Journal of Management Information Systems, vol. 26, no. 1, pp. 73-100, 2009. Cited on page(s): 3

[25] A. Goel and D. Joyner, "Design of an online course on knowledge-based ai," in Proceedings of the AAAI Conference on Artificial Intelligence, vol. 30, no. 1, 2016. Cited on page(s): 3

[26] O. Noroozi, A. Weinberger, H. J. Biemans, M. Mulder, and M. Chizari, "Facilitating argumentative knowledge construction through a transactive discussion script in cscl," Computers \& Education, vol. 61, pp. 59-76, 2013. Cited on page(s): 3

[27] P. Dillenbourg, S. Lemaignan, M. Sangin, N. Nova, and G. Molinari, "The symmetry of partner modelling," International Journal of Computer-Supported Collaborative Learning, vol. 11, no. 2, pp. 227-253, 2016. Cited on page(s): 3 
[28] S. Frost and G. McCalla, "A planning algorithm to support learning in open-ended, unstructured environments," International Journal of Artificial Intelligence in Education, Oct 2020. [Online]. Available: https://doi.org/10.1007/s40593-020-00221-3 Cited on page $(s): 3$

[29] J. Kay and B. Kummerfeld, "From data to personal user models for life-long, life-wide learners," British Journal of Educational Technology, vol. 50, no. 6, pp. 2871-2884, 2019. [Online]. Available: https://bera-journals.onlinelibrary.wiley.com/doi/abs/10.1111/ bjet.12878 Cited on page(s): 3

[30] D. Gale and L. S. Shapley, "College admissions and the stability of marriage," The American Mathematical Monthly, vol. 69, no. 1, pp. 9-15, 1962. Cited on page(s): 3

[31] The National Resident Matching Program (NRMP), 2021 (accessed 2021-02-1), https://www. nrmp.org/matching-algorithm/. Cited on page(s): 3

[32] K. Iwama and S. Miyazaki, "A survey of the stable marriage problem and its variants," in International conference on informatics education and research for knowledge-circulating society (ICKS 2008). IEEE, 2008, pp. 131-136. Cited on page(s): 3

[33] H. Jeong, C. E. Hmelo-Silver, and K. Jo, "Ten years of computer-supported collaborative learning: A meta-analysis of cscl in stem education during 2005-2014," Educational research review, vol. 28, p. 100284, 2019. Cited on page(s): 3

[34] A. Stewart and S. K. D'Mello, "Connecting the dots towards collaborative aied: Linking group makeup to process to learning," in International Conference on Artificial Intelligence in Education. Springer, 2018, pp. 545-556. Cited on page(s): 3

[35] T. Jadin, T. Gnambs, and B. Batinic, "Personality traits and knowledge sharing in online communities," Computers in Human Behavior, vol. 29, no. 1, pp. 210-216, 2013. Cited on page(s): 3

[36] Z. Mehennaoui, Y. Lafifi, H. Seridi, and A. Boudria, "A new approach for grouping learners in cscl systems," in 2014 International Conference on Multimedia Computing and Systems (ICMCS). IEEE, 2014, pp. 628-632. Cited on page(s): 3

[37] V. D. Yannibelli, M. G. Armentano, F. D. Berdun, and A. A. Amandi, "A steady-state evolutionary algorithm for building collaborative learning teams in educational environments considering the understanding levels and interest levels of the students," 2016. Cited on page(s): 3

[38] A. Ounnas, H. Davis, and D. Millard, "A framework for semantic group formation," in 2008 Eighth IEEE international conference on advanced learning technologies. IEEE, 2008, pp. 34-38. Cited on page(s): 3

[39] G. Makransky, P. Wismer, and R. E. Mayer, "A gender matching effect in learning with pedagogical agents in an immersive virtual reality science simulation," Journal of Computer Assisted Learning, vol. 35, no. 3, pp. 349-358, 2019. Cited on page(s): 3

[40] W. M. Cruz and S. Isotani, "Group formation algorithms in collaborative learning contexts: A systematic mapping of the literature," in CYTED-RITOS International Workshop on Groupware. Springer, 2014, pp. 199-214. Cited on page(s): 3 
[41] J. Greer, "Children and internet pornography: a moral panic, a salvation for censors and trojan horse for government colonisation of the digital frontier," Revisiting Moral Panics (Moral Panics in Theory and Practice), pp. 137-148, 2016. Cited on page(s): 3

[42] J. E. Greer, G. Mccalla, J. A. Collins, V. S. Kumar, P. Meagher, and J. Vassileva, "Supporting peer help and collaboration in distributed workplace environments," International Journal of Artificial Intelligence in Education (IJAIED), vol. 9, pp. 159-177, 1998. Cited on page(s): 3

[43] X. Xu, L. Zhang, and Q. Wan, "A variation coefficient similarity measure and its application in emergency group decision-making," Systems Engineering Procedia, vol. 5, pp. 119-124, 2012, safety and Emergency Systems Engineering. [Online]. Available: https://www.sciencedirect.com/science/article/pii/S2211381912000628 Cited on page(s): 6

[44] R. Shi, K. N. Ngan, and S. Li, "Jaccard index compensation for object segmentation evaluation," in 2014 IEEE international conference on image processing (ICIP). IEEE, 2014, pp. 4457-4461. Cited on page(s): 9

[45] Y. Pang, F. Xiao, H. Wang, and X. Xue, "A clustering-based grouping model for enhancing collaborative learning," in 2014 13th International Conference on Machine Learning and Applications. IEEE, 2014, pp. 562-567. Cited on page(s): 9 